\title{
Hyper-Miniaturisation of Monodisperse Janus Hydrogel Beads with Magnetic Anisotropy Based on Coagulation of $\mathrm{Fe}_{3} \mathrm{O}_{4}$ Nanoparticles
}

\author{
Kyouhei Aketagawa, Hirotada Hirama*, Toru Torii \\ Department of Human and Engineered Environmental Studies, Graduate School of Frontier Sciences, \\ The University of Tokyo, Ka-shiwa-shi, Japan \\ Email: "hhirama@dt.k.u-tokyo.ac.jp
}

Received May 8, 2013; revised June 9, 2013; accepted June 21, 2013

Copyright (C) 2013 Kyouhei Aketagawa et al. This is an open access article distributed under the Creative Commons Attribution License, which permits unrestricted use, distribution, and reproduction in any medium, provided the original work is properly cited.

\begin{abstract}
We describe a simple and robust technique for fabricating monodisperse Janus hydrogel beads with magnetic anisotropy smaller than the microchannel used to produce them.
\end{abstract}

Keywords: Magnetic Janus Particles; Microchannels; Calcium Alginate Gel; Monodisperse; Osmotic Pressure; Coagulation

\section{Introduction}

Janus particles are anisotropic microparticles with hemispherically distinct geometries. Janus particles with magnetic anisotropy have attracted much attention and can be used in electronic paper [1,2], rheological probes [3], self-assembly [4-6] and biomedical applications [7-9] because they can be rotated and precisely manipulated by an external magnetic field [1-9]. The mono-dispersity of the Janus particles enhances the performance of such applications. Therefore, many studies have focused on monodisperse biphasic droplet formation techniques using microchannels for the fabrication of Janus particles with magnetic anisotropy $[2,6-8]$.

With respect to electronic paper using Janus particles, a decrease in the thickness of the electronic paper can allow for operation at a low electric voltage, and the thickness depends on the size of the particles [10]. For cell encapsulation, the cell is strongly affected by the size of the capsules [11]. Therefore control of the particle size is needed. However, there are limits to the minimum monodisperse biphasic droplet size that can be fabricated using microchannels because the microchannel size has a dominant effect on the monodisperse droplet size [12-15] and the risk of clogging a smaller microchannel is higher $[2,16]$. Moreover, convective mixing across the interface

\footnotetext{
"Corresponding author.
}

inside the small biphasic droplets is enhanced, leading to fabrication of Janus particles with a blurred boundary or completely homogeneous particles $[3,17,18]$. It is therefore difficult to fabricate small Janus particles with magnetic anisotropy.

In our previous work, a new shrinkage-gelation technique was developed in which small monodisperse alginate-inorganic composite particles were fabricated from larger sodium alginate droplets containing inorganic nanoparticles [19-21]. In this method, monodisperse sodium alginate droplets containing inorganic nanoparticles formed in microchannels are placed at the interface between a high-viscosity oil with a polymeric surfactant and a high-strength hydrogel containing calcium chloride. Because there is a difference in osmotic pressure between the alginate droplets and the $\mathrm{Ca}^{2+}$-loaded hydrogel, water molecules in the droplets transfer to the hydrogel, [22] resulting in the shrinkage of the droplets. The droplets then solidify due to transfer of calcium ions from the $\mathrm{Ca}^{2+}$-loaded hydrogel to the droplets $[23,24]$.

In this study, we report a simple and robust technique for fabricating monodisperse Janus hydrogel beads with magnetic anisotropy at a size that is smaller than the microchannel used to produce them based on the coagulation of $\mathrm{Fe}_{3} \mathrm{O}_{4}$ nanoparticles and the shrinkage-gelation technique, which can avoid the problems of clogging microchannels and fabricating Janus particles with a blurred boundary due to convective mixing. 


\section{Experimental}

We fabricated a microchannel made of polydimethylsiloxane (PDMS) via multilayer photolithography [25]. As shown in Figure 1, the microchannel consisted of a Yshaped dispersed phase channel measuring $100 \mu \mathrm{m}$ in width and depth and a sheath-flow junction. The sheathflow structure was composed of two continuous phase channels measuring $100 \mu \mathrm{m}$ in width and depth and a channel measuring $200 \mu \mathrm{m}$ in width and depth.

We first prepared a $\mathrm{Ca}^{2+}$-loaded agarose gel plate. A solution of $2 \mathrm{w} / \mathrm{v} \%$ agarose $\mathrm{S}$ (Wako Pure Chemical Industries, Ltd., Japan) was prepared in calcium chloride aqueous solution $(0.5,1.0,1.5$ or $2.0 \mathrm{~mol} / \mathrm{L})$ at approximately $95^{\circ} \mathrm{C}$, and the solution was poured into a polystyrene dish and solidified at $25^{\circ} \mathrm{C}$. Subsequently, an oil/ hydrogel interface was formed by pouring $0.8 \mathrm{~mL} /$ well of sunflower oil (Showa Sangyo Co., Ltd., Japan) supple

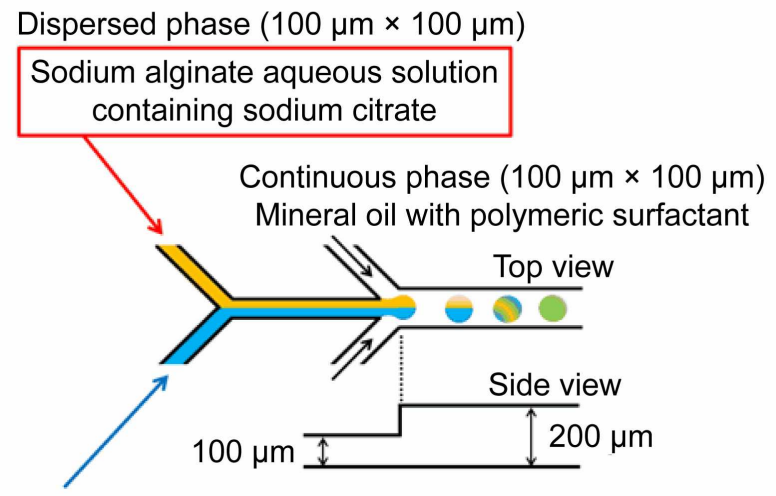

Dispersed phase $(100 \mu \mathrm{m} \times 100 \mu \mathrm{m})$

Sodium alginate aqueous solution containing $\mathrm{Fe}_{3} \mathrm{O}_{4}$ nanoparticles

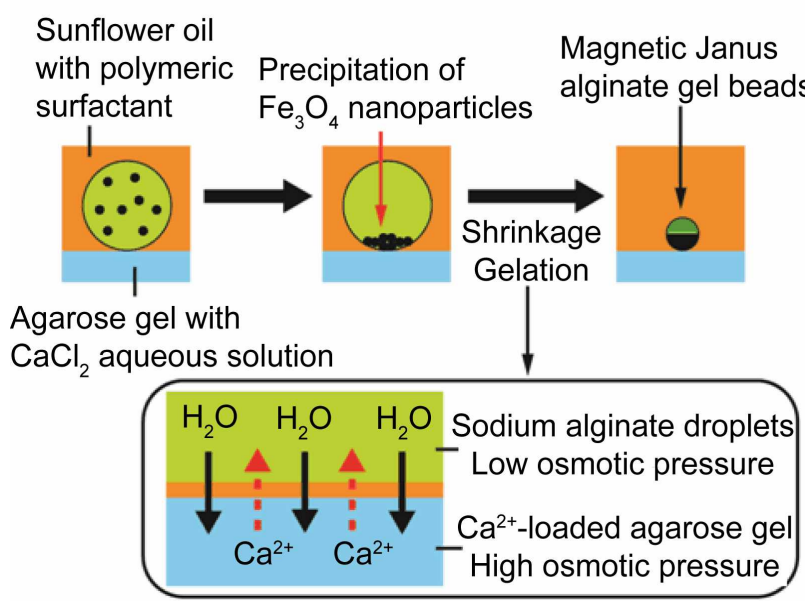

Figure 1. Fabrication method of small Janus hydrogel beads with magnetic anisotropy. (Top) Two-phase parallel stream forms biphasic droplets. (Bottom) Small magnetic Janus alginate gel beads are fabricated on the $\mathrm{Ca}^{2+}$-loaded agarose gel plate. mented with polymeric surfactant (polyglycerol esters of fatty acids, SY GLYSTER CRS-75, Sakamoto Yakuhin Kogyo Co., Ltd, Japan) onto the agarose gel plate.

In the method presented here, we induce coagulation by electrolyte addition during the fabrication of Janus hydrogel beads with magnetic anisotropy. When $\mathrm{Fe}_{3} \mathrm{O}_{4}$ nanoparticles are suspended in an aqueous solution, electric double layers form spontaneously around the nanoparticles and a repulsion barrier generated by the electric double layers prevents the nanoparticles from aggregating [26]. Because the thickness of the double layers is inversely proportional to the concentration of ions in the aqueous solution, the repulsion between the nanoparticles decreases as the concentration of electrolyte added in the aqueous solution increases, resulting in aggregation of the nanoparticles by the van der Waals force between them [26].

Hofmeister examined the concentrations of the thirteen types of sodium salts necessary to precipitate colloidal ferric oxide suspended in an aqueous solution and discovered that sodium citrate can aggregate and precipitate ferric oxide at the lowest concentration [27]. Based on these results, we examined the concentration of sodium citrate necessary to precipitate $\mathrm{Fe}_{3} \mathrm{O}_{4}$ nanoparticles measuring $10 \mathrm{~nm}$ in mean diameter (Ferrotec Corporation, Japan) suspended in water. In the case that the density of $\mathrm{Fe}_{3} \mathrm{O}_{4}$ nanoparticles was $1.0 \mathrm{w} / \mathrm{v} \%$, the minimum concentration of sodium citrate for completely precipitating $\mathrm{Fe}_{3} \mathrm{O}_{4}$ nanoparticles was approximately 0.01 $\mathrm{mol} / \mathrm{L}$

The fabrication method for small Janus hydrogel beads with magnetic anisotropy is shown in Figure 1. A 1.0 $\mathrm{w} / \mathrm{w} \%$ sodium alginate aqueous solution containing 0.02 $\mathrm{mol} / \mathrm{L}$ sodium citrate and a $1.0 \mathrm{w} / \mathrm{w} \%$ sodium alginate aqueous solution containing $1.6 \mathrm{w} / \mathrm{v} \%$ paramagnetic $\mathrm{Fe}_{3} \mathrm{O}_{4}$ nanoparticles were injected separately through two inlets resulting in a two-phase parallel stream in the microchannel due to the low Reynolds number. This stream then entered a continuous flow of mineral oil (SigmaAldrich Co., Ltd., USA) with a polymeric surfactant (SY GLYSTER CRS-75), and biphasic droplets were formed. Convective mixing immediately occurred across the interface inside the biphasic droplets. Next, sodium alginate droplets containing both $\mathrm{Fe}_{3} \mathrm{O}_{4}$ nanoparticles and sodium citrate were formed. The $\mathrm{Fe}_{3} \mathrm{O}_{4}$ nanoparticles aggregated in the presence of sodium citrate and electrolyte, and they precipitated at the bottom of the droplets (Figure 1, bottom). The droplets were placed on the $\mathrm{Ca}^{2+}$-loaded agarose gel plate, resulting in fabrication of small Janus alginate gel beads with magnetic anisotropy.

\section{Results and Discussion}

Figure 2(a) shows the monodisperse sodium alginate 
(a)

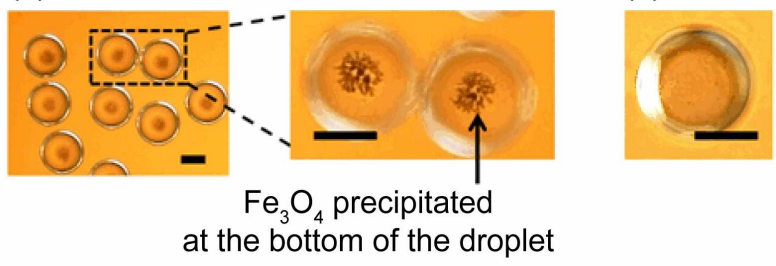

(c)

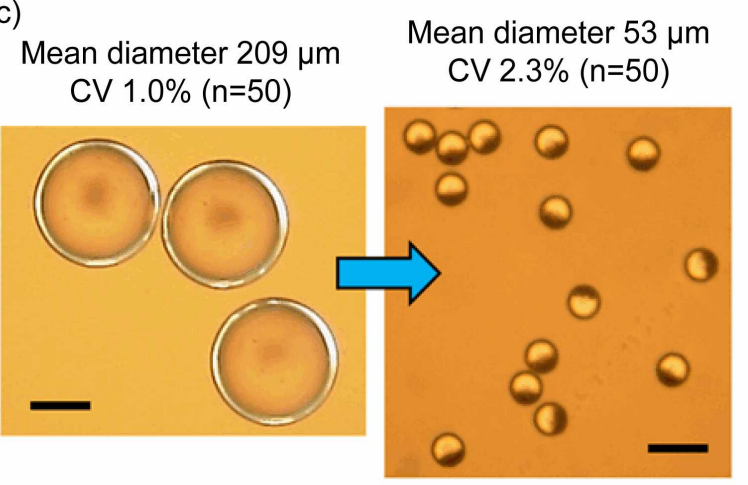

(d)

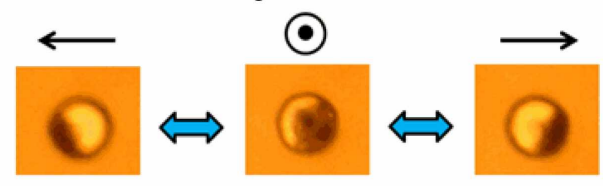

Figure 2. (a) $\mathrm{Fe}_{3} \mathrm{O}_{4}$ nanoparticles immediately aggregated and precipitated at the bottom of the sodium alginate droplets containing sodium citrate; (b) $\mathrm{No}^{\mathrm{Fe}} \mathrm{O}_{3}$ nanoparticles aggregated inside the sodium alginate droplets without sodium citrate; (c) The alginate droplets shrank and solidified with $\mathrm{Fe}_{3} \mathrm{O}_{4}$ nanoparticles precipitated at the bottom of them, resulting in fabrication of small Janus hydrogel beads with magnetic anisotropy. " $n$ " indicates the measured number of droplets/particles; (d) The fabricated magnetic Janus alginate gel beads rotated with an external magnetic field, which is shown as the direction of the arrows. Scale bar is $100 \mu \mathrm{m}$.

droplets containing both $\mathrm{Fe}_{3} \mathrm{O}_{4}$ nanoparticles and sodium citrate formed using the microchannel. Inside the drop lets, $\mathrm{Fe}_{3} \mathrm{O}_{4}$ nanoparticles promptly aggregated and precipitated at the bottom of the droplet because the electric double layers enclosing $\mathrm{Fe}_{3} \mathrm{O}_{4}$ nanoparticles became thin due to the sodium citrate and they nanoparticles were attracted to each other by strong van der Waals force [26]. In sodium alginate droplets without sodium citrate, no aggregation and precipitation of $\mathrm{Fe}_{3} \mathrm{O}_{4}$ nanoparticles occurred (Figure 2(b)). By placing the sodium alginate droplets containing both $\mathrm{Fe}_{3} \mathrm{O}_{4}$ nanoparticles and sodium citrate on the $\mathrm{Ca}^{2+}$-loaded agarose gel plate, the droplets shrank with the $\mathrm{Fe}_{3} \mathrm{O}_{4}$ nanoparticles that had precipitated at the bottom and solidified. As a result, small magnetic Janus alginate gel beads were fabricated from larger original droplets (Figure 2(c)). The coefficient of variation $(\mathrm{CV})$ of the magnetic Janus alginate gel beads was less than 5\%; therefore, monodispersity was maintained after shrinkage. The fabricated Janus gel beads had orientational interactions and rotated without physical translation under an external magnetic field induced by a permanent magnet $(60 \mathrm{mT})$ (Figure 2(d)). With respect to the risk of blurring the boundary by the convective mixing inside the biphasic droplets during their solidification, phase separation in the original droplets was induced by aggregation and precipitation of $\mathrm{Fe}_{3} \mathrm{O}_{4}$ nanoparticles; therefore, the risk of clogging could be eliminated. With respect to microchannel clogging, Wyss reports that the clogging depends on the number of particles that pass through a channel; therefore, clogging occurs more rapidly as the particle volume fraction is increased [16]. In our method, the density of $\mathrm{Fe}_{3} \mathrm{O}_{4}$ nanoparticles increased due to shrinkage of the sodium alginate droplets with the nanoparticles. Therefore, the initial density of the nanoparticles in the sodium alginate aqueous solution injected into the microchannel could be low enough to avoid clogging the microchannels.

We examined the relationship between the shrinkage factor and the difference in osmotic pressure between the sodium alginate droplets and the calcium chloride aqueous solution contained in the agarose gel plate. The shrinkage factor was defined as the volume change of the droplets.

$$
\text { Shrinkage factor }(\%)=\left(\mathrm{V}_{\mathrm{o}}-\mathrm{V}_{\mathrm{r}}\right) / \mathrm{V}_{\mathrm{o}}
$$

$\mathrm{V}_{\mathrm{o}}$ : Volume of the original droplets;

$\mathrm{V}_{\mathrm{r}}$ : Volume of resulting magnetic Janus hydrogel beads.

As shown in Figure 3, the shrinkage factor was saturated at approximately $98.4 \%$ with a large difference in

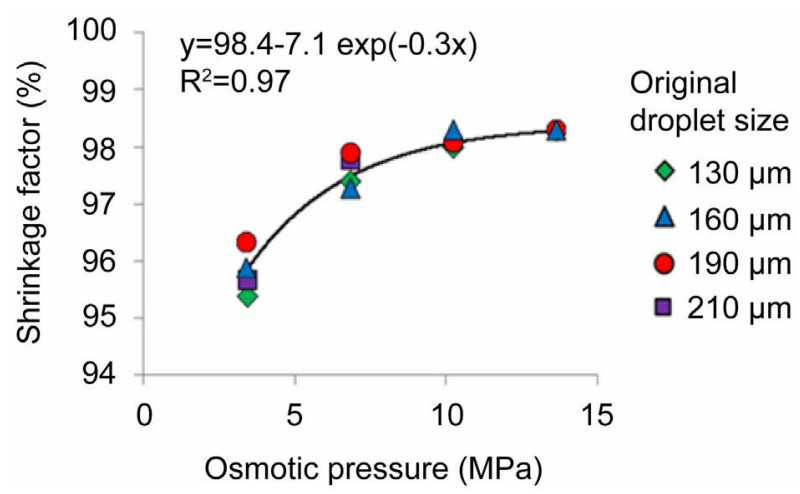

Figure 3. The relationship between the shrinkage factor and the difference in osmotic pressure between the sodium alginate droplets and the calcium chloride aqueous solution. The data were acquired from the original droplets measureing 130, 160, 190 and $210 \mu \mathrm{m}$ in mean diameter $(\mathrm{CV}<5 \%$, $\mathbf{n}=\mathbf{3 0})$. 
osmotic pressure. The regression line was approximated with a first order lag model, showing a strong correlation between the shrinkage factor and the osmotic pressure. We can therefore predict the size of magnetic Janus alginate gel beads by the original droplet size and the difference in osmotic pressure between the sodium alginate droplets and the calcium chloride aqueous solution contained in the agarose gel plate.

\section{Conclusion}

This technique allows for the fabrication of the optimal size of monodisperse Janus hydrogel beads with magnetic anisotropy corresponding to various applications stably and easily.

\section{REFERENCES}

[1] A. Ghosh, N. K. Sheridon and P. Fischer, "Voltage-Controllable Magnetic Composite Based on Multifunctional Polyethylene Microparticles," Small, Vol. 4, No. 11, 2008, pp. 1956-1958. doi:10.1002/smll.200701301

[2] S. N. Yin, C. F. Wang, Z. Y. Yu, J. Wang, S. S. Liu and S. Chen, "Versatile Bifunctional Magnetic-Fluorescent Responsive Janus Supraballs towards the Flexible Bead Display," Advanced Materials, Vol. 23, No. 26, 2011, pp. 2915-2919.

[3] R. K. Shah, J. Kim and D. A. Weitz, "Janus Supraparticles by Induced Phase Separation of Nanoparticles in Droplets," Advanced Materials, Vol. 21, No. 19, 2009, pp. 1949-1953.

[4] N. Zhao and M. Y. Gao, "Magnetic Janus Particles Prepared by a Flame Synthetic Approach: Synthesis, Characterizations and Properties," Advanced Materials, Vol. 21, No. 2, 2009, pp. 184-187.

[5] A. K. F. Dyab, M. Ozmen, M. Ersoz and V. N. Paunov, "Fabrication of Novel Anisotropic Magnetic Microparticles," Journal of Materials Chemistry, Vol. 19, No. 21, 2009, pp. 3475-3481. doi:10.1039/b814971m

[6] K. P. Yuet, D. K. Hwang, R. Haghgooie and P. S. Doyle, "Multifunctional Superparamagnetic Janus Particles," Langmuir, Vol. 26, No. 6, 2010, pp. 4281-4287. doi:10.1021/la903348s

[7] L. B. Zhao, L. Pan, K. Zhang, S. S. Guo, W. Liu, Y. Wang, Y. Chen, X. Z. Zhao and H. L. W. Chan, "Generation of Janus Alginate Hydrogel Particles with Magnetic Anisotropy for Cell Encapsulation," Lab on a Chip, Vol. 9, No. 20, 2009, pp. 2981-2986. doi:10.1039/b907478c

[8] Y. J. Zhao, H. C. Shum, H. S. Chen, L. L. A. Adams, Z. Z. $\mathrm{Gu}$ and D. A. Weitz, "Microfluidic Generation of Multifunctional Quantum Dot Barcode Particles," Journal of the American Chemical Society, Vol. 133, No. 23, 2011, pp. 8790-8793. doi:10.1021/ja200729w

[9] C. H. Chen, A. R. Abate, D. Y. Lee, E. M. Terentjev and D. A. Weitz, "Microfluidic Assembly of Magnetic Hydrogel Particles with Uniformly Anisotropic Structure," Advanced Materials, Vol. 21, No. 31, 2009, pp. 32013204.
[10] Y. Lee, S. Lee, J.-B. Kwak, S. Kim, H.-Y. Cha, C. H. Lee, C. S. Lee and H.-S. Lee, "Friction Effect on the Rotation of Bichromal Ball in a Monolayer Rotating Ball Display," Journal of Applied Physics, Vol. 108, 2010, Article ID: 102812. doi:10.1063/1.3511710

[11] W. H. Tan and S. Takeuchi, "Monodisperse Alginate Hydrogel Microbeads for Cell Encapsulation," Advanced Materials, Vol. 19, No. 18, 2007, pp. 2696-2701. doi:10.1002/adma.200700433

[12] T. Nisisako and T. Torii, "Formation of Biphasic Janus Droplets in a Microfabricated Channel for the Synthesis of Shape-Controlled Polymer Microparticles," Advanced Materials, Vol. 19, No. 11, 2007, pp. 1489-1493.

[13] D. R. Link, E. Grasland-Mongrain, A. Duri, F. Sarrazin, Z. D. Cheng, G. Cristobal, M. Marquez and D. A. Weitz, "Electric Control of Droplets in Microfluidic Devices," Angewandte Chemie-International Edition, Vol. 45, No. 16, 2006, pp. 2556-2560. doi:10.1002/anie.200503540

[14] H. Kim, D. W. Luo, D. Link, D. A. Weitz, M. Marquez, and Z. D. Cheng, "Controlled Production of Emulsion Drops Using an Electric Field in a Flow-Focusing Microfluidic Device," Applied Physics Letters, Vol. 91, No. 13, 2007, Article ID: 133106. doi:10.1063/1.2790785

[15] T. Nisisako, T. Torii and T. Higuchi, "Novel MicroreacTors for Functional Polymer Beads," Chemical Engineering Journal, Vol. 101, No. 1-3, 2004, pp. 23-29. doi:10.1016/j.cej.2003.11.019

[16] H. M. Wyss, D. L. Blair, J. F. Morris, H. A. Stone and D. A. Weitz, "Mechanism for Clogging of Microchannels," Physical Review E, Vol. 74, No. 6, 2006, Article ID: 061402. doi:10.1103/PhysRevE.74.061402

[17] T. Nisisako, T. Torii, T. Takahashi and Y. Takizawa, "Synthesis of Monodisperse Bicolored Janus Particles with Electrical Anisotropy Using a Microfluidic Co-Flow System," Advanced Materials, Vol. 18, No. 9, 2006, pp. 11521156. doi:10.1002/adma.200502431

[18] S. Ahn, D. W. Kim, Y. W. Kim and J. Y. Yoo, "Generation of Janus Droplets for Enhanced Mixing in Microfluidics," International Journal of Precision Engineering and Manufacturing, Vol. 11, No. 5, 2010, pp. 799-802. doi:10.1007/s12541-010-0095-z

[19] H. Hirama, T. Kambe, K. Aketagawa, T. Ota, H. Moriguchi and T. Torii, "Hyper Alginate Gel Microbead Formation by Molecular Diffusion at the Hydrogel/Droplet Interface," Langmuir, Vol. 29, No. 2, 2013, pp. 519-524. doi:10.1021/la303827u

[20] K. Aketagawa, H. Hirama, H. Moriguchi and T. Torii, "Fabrication of Titania Microspheres Using Alginate Microdroplets on an Oil/Hydrogel Interface," The 15th International Conference on Miniaturized Systems for Chemistry and Life Sciences, Seattle, 2-6 October 2011, pp. 1615-1617.

[21] K. Aketagawa, H. Hirama, H. Moriguchi and T. Torii, "Janus Hydrogel Beads for Electronic Paper Using Shrinkage-Gelation Technique," The 16th International Conference on Miniaturized Systems for Chemistry and Life Sciences, Okinawa, 28 October-1 November 2012, pp. 890-892. 
[22] L. X. Wen and K. D. Papadopoulos, "Effects of Osmotic Pressure on Water Transport in W-1/O/W-2 Emulsions," Journal of Colloid and Interface Science, Vol. 235, No. 2, 2001, pp. 398-404. doi:10.1006/jicis.2000.7384

[23] J. Cheng, J. F. Chen, M. Zhao, Q. Luo, L. X. Wen and K. D. Papadopoulos, "Transport of Ions through the Oil Phase of W-1/O/W-2 Double Emulsions," Journal of Colloid and Interface Science, Vol. 305, No. 1, 2007, pp. 175-182. doi:10.1016/j.jcis.2006.09.055

[24] G. Talei Franzesi, B. Ni, Y. Ling and A. Khademhosseini, "A Controlled-Release Strategy for the Generation of Cross-Linked Hydrogel Microstructures," Journal of the American Chemical Society, Vol. 128, No. 47, 2006, pp. 15064-15065.
[25] D. Saeki, S. Sugiura, T. Kanamori, S. Sato and S. Ichikawa, "Microfluidic Preparation of Water-in-Oil-in-Water Emulsions with an Ultra-Thin Oil Phase Layer," Lab on a Chip, Vol. 10, No. 3, 2010, pp. 357-362. doi:10.1039/b916318b

[26] R. J. Hunter, "Foundations of Colloid Science," Oxford University Press, Oxford, 2001.

[27] W. Kunz, J. Henle and B. W. Ninham, “'Zur Lehre von der Wirkung der Salze' (about the Science of the Effect of Salts): Franz Hofmeister's Historical Papers," Current Opinion in Colloid \& Interface Science, Vol. 9, No. 1, 2004, pp. 19-37. doi:10.1016/j.cocis.2004.05.005 